\title{
Effects of young extracellular matrix on the biological characteristics of aged tendon stem cells
}

\author{
Dapeng Jiang ${ }^{1, A, C, D, F}, B 0 X^{2}, B, C, E$, Peng Gao ${ }^{2, C-F}$ \\ 1 Department of Urology, Shanghai Children's Medical Center, Shanghai Jiao Tong University School of Medicine, China \\ ${ }^{2}$ Department of Pediatric Surgery, Second Affiliated Hospital of Harbin Medical University, China \\ A - research concept and design; $\mathrm{B}$ - collection and/or assembly of data; $\mathrm{C}$ - data analysis and interpretation; \\ $D$ - writing the article; $E$ - critical revision of the article; $F$ - final approval of the article
}

\section{Address for correspondence \\ Dapeng Jiang \\ E-mail:jdp509@163.com \\ Funding sources \\ This work was supported by grants from the National Natural Sciences Foundation of China (No. 30901516, 81472085).}

\section{Conflict of interest}

None declared

Received on March 19, 2016

Reviewed on May 30, 2016

Accepted on June 26, 2017

\begin{abstract}
Background. Age-related changes in the properties of tendon stem cells (TSC) may play a role in the progressive degeneration and increased risk of injury to tendon tissue. Recent reports have demonstrated that a decellularized extracellular matrix (DECM) can provide an appropriate niche to maintain the proliferation and differentiation capacity of adult stem cells.
\end{abstract}

Objectives. We investigated the biological effects of DECM obtained from young TSCs on the proliferation, stemness, senescence, and differentiation of the aged TSCS.

Material and methods. Tendon stem cells were isolated from rat patellar tendons and the DECM was collected. The proliferative capacity, $\beta$-galactosidase activity, stem cell marker expression, and tenogenic differentiation potential of TSCs were assessed.

Results. Our results showed that DECM from young TSCs enhanced the proliferation and tenogenic differentiation of aged TSCS. Moreover, the senescence-associated $\beta$-galactosidase activity of aged TSCs was decreased by young DECM. After being cultured on the young DECM, the expression of stem cell markers by aged TSCs was more extensive. The young DECM preserved stem cell properties of aged TSCS.

Conclusions. Taken together, the impaired capacity of aged TSCs can be rejuvenated by exposure to young DECM. The positive results in our study suggest that young TSC-derived DECM may provide a novel approach for the prevention and treatment of age-dependent tendon disorders.

Key words: proliferation, aging, extracellular matrix, differentiation, tendon

DOI

$10.17219 /$ acem $/ 75503$

\section{Copyright}

Copyright by Author(s)

This is an article distributed under the terms of the

Creative Commons Attribution Non-Commercial License

(http://creativecommons.org/licenses/by-nc-nd/4.0/) 
Tendon disorders, such as injury and tendinopathy, pose a critical challenge for sports medicine. It was suggested that the rupture of a tendon often occurs in aged tendons which are weakened by overuse or degeneration. ${ }^{1}$ Age-related changes in the structural and functional properties of tendon tissue contribute to the progressive degeneration and an increased risk of injury. ${ }^{2,3}$ Tendon injuries and tendinopathy cause physical frailty in old age with a general reduction in quality of life. There are changes in the number and activity of tendon cells observed with aging. ${ }^{4,5}$ Moreover, aging has been postulated to exert a negative effect on the proliferation and synthesis of tenocytes. ${ }^{6}$ Thus, tendon injuries in a geriatric patient are believed to regenerate more slowly.

The ultimate goal of treating tendon injury is to regenerate tissues. Cells in tendon tissue that produce and organize extracellular matrix (ECM) are very important in maintaining the homeostasis of tendons. More recently, it was reported that tendon tissues contain a minor population of cells with stem cell properties, called tendon stem cells (TSCs). ${ }^{7,8}$ Moreover, the application of these stem cells has been considered a promising approach for the restoration of structure and function after tendon injury. ${ }^{9,10}$ However, the negative effects of increased age on the properties of TSCs and tenocytes have also been reported. ${ }^{5}$ These age-related changes in TSCs would seriously affect the regeneration of tendons.

Studies have demonstrated that TSCs need a specific microenvironment, the so-called stem cell niche, in order to keep their stemness or to orient tenogenic differentiation. ${ }^{10,11}$ It has been reported that the structure and ECM protein expression levels in aged tendons are different from those found in young ones. ${ }^{2}$ Recent studies have also demonstrated that the decellularized stem cell matrix can provide an appropriate niche in which adult stem cells can greatly expand, delay replicative senescence and restore their differentiation capacity. ${ }^{12,13}$ We hypothesize that decellularized extracellular matrix (DECM) derived from young TSCs can modulate the properties of aged TSCs. Based on this hypothesis, the present study was designed to investigate the biological effect of DECM of young TSCs on the proliferation, stemness, senescence, and differentiation of aged TSCs.

\section{Material and methods}

\section{Isolation and culture of rat}

All experiments in this study were performed in accordance with the guidelines for animal research from the National Institutes of Health (Bethesda, USA) and were carried out under the Rules and Regulations of the Animal Care and Use Committee at Shanghai Jiao Tong University School of Medicine, China. Ten young (2-month-old) and 10 old (24-month-old) Sprague-Dawley male rats were used for the isolation of TSCs. Tendon stem cells were isolated from rat patellar tendons. The procedures for the isolation and culture of TSCs have been established. ${ }^{8}$ The midsubstance of the patellar tendon was removed, cut into small sections and minced. The tissues were digested with type I collagenase ( $3 \mathrm{mg} / \mathrm{mL}$; Sigma-Aldrich, St. Louis, USA) and dispase ( $4 \mathrm{mg} / \mathrm{mL}$; Stem Cell Technologies, Vancouver, Canada) at $37^{\circ} \mathrm{C}$ for $1 \mathrm{~h}$. The fragments were then passed through a 70-mm cell strainer (Becton Dickinson, Tokyo, Japan) to yield a single-cell suspension. The isolated cells were resuspended in Dulbecco's Modified Eagle Medium (DMEM), 10\% fetal bovine serum (FBS), and 1\% penicillin and streptomycin. After 8-10 days in culture, TSCs formed colonies on the culture plates and were collected through the local application of trypsin and mixed together as passage 0 . The stem cell identities of TSCs were routinely confirmed by the expression of stem-cellrelated surface markers, clonogenicity and multilineage differentiation potentials before being used in this study.

\section{Preparation of decellularized extracellular matrix generated by young or aged tendon stem cells}

Decellularized extracellular matrix was obtained using the previously described method. ${ }^{14}$ Six-well plates were precoated with $0.2 \%$ gelatin (Sigma-Aldrich) at $37^{\circ} \mathrm{C}$ for $1 \mathrm{~h}$ and young or old TSCs at passage 2 were plated at a density of 4000 cells $/ \mathrm{cm}^{2}$. L-ascorbic acid phosphate $(50 \mathrm{mg} / \mathrm{mL})$ (Sigma-Aldrich) was added to the medium for 8 days after the cells reached $90 \%$ confluence. The deposited ECM by TSCs was incubated with $0.5 \%$ Triton X-100 (Sigma-Aldrich) containing $20 \mathrm{mM}$ of ammonium hydroxide for $5 \mathrm{~min}$ at $37^{\circ} \mathrm{C}$ followed by treatment with $100 \mathrm{U} / \mathrm{mL}$ of DNase at room temperature for $1 \mathrm{~h}$. Then, the matrix was stored in phosphate-buffered saline (PBS) containing antibiotics at $4^{\circ} \mathrm{C}$ for subsequent cell culture experiments.

\section{Tendon stem cells grown on decellularized extracellular matrix}

Aged and young TSCs at passage 2 were plated at a density of 3000 cells $/ \mathrm{cm}^{2}$ in 6 -well plates coated with or without DECM. There were 4 groups, including a young TSC group, an aged TSC group, an aged TSC + aged DECM group, and an aged TSC + young DECM group. The TSCs were cultured in DMEM containing 10\% FBS and antibiotics for 7 days at $37^{\circ} \mathrm{C}$ under $5 \% \mathrm{CO}_{2}$. The medium was changed every 3 days.

\section{Cell proliferation assay}

To assess the effect of DECM on the proliferation of aged TSCs, cell numbers were counted and the population doubling time (PDT) of each group was estimated as previously described. ${ }^{8}$ 


\section{Senescence-associated $\beta$-galactosidase activity assay}

The senescence-associated $\beta$-galactosidase activity was determined as described previously. ${ }^{15}$ A sample of $50 \mathrm{mg}$ of protein from TSCs in each group (HT) and TDSCs (CI) at P5 was used to assess the senescence-associated $\beta$-galactosidase activity by using a Pierce Mammalian $\beta$-Galactosidase Assay Kit (Thermo Scientific, Inc., Rockford, USA). Absorbances were measured at $405 \mathrm{~nm}$ using a plate-reading spectrophotometer.

\section{Immunostaining of stem cell markers}

Immunocytochemistry was used to examine the following stem cell markers: octamer-binding transcription factor 4 (Oct-4) and stage-specific embryonic antigen-4 (SSEA-4). The TSCs were fixed with freshly prepared $4 \%$ paraformaldehyde for $30 \mathrm{~min}$ and then permeabilized with 0.1\% Triton X-100 (Sigma-Aldrich) for $20 \mathrm{~min}$ at room temperature. After being washed with PBS 3 times, they were incubated with the primary antibody monoclonal mouse anti-Oct-4 (1:350). After the cells were washed with PBS, Cy3-conjugated secondary antibody (1:500) was applied for $1 \mathrm{~h}$ at room temperature in the dark. For the staining of SSEA-4, the cells were fixed with $4 \%$ paraformaldehyde. Cells were then incubated with mouse anti-SSEA-4 antibody (1:350) for $2 \mathrm{~h}$ at room temperature. After washing, a secondary antibody (1:500) conjugated with fluorescein isothiocyanate (FITC) was applied for $1 \mathrm{~h}$ at room temperature in a darkened humidified chamber. Finally, the samples were washed with PBS and mounted in nuclear staining reagent Hoechst fluorochrome 33342 (1 mg/mL; Sigma-Aldrich). Each tissue section was observed under a fluorescence microscope. Twenty-five views from all 6 samples per group were obtained. Fifty cells were counted in 1 view and the percentage of stem cell markerpositive cells was analyzed for each staining.

\section{Expression of tenogenic markers}

At day 7, the cells were harvested and the expression of tenogenic markers, including tenomodulin (Tnmd) and scleraxis $(\mathrm{Scx})$, were evaluated using quantitative reverse transcription polymerase chain reaction (qRT-PCR). The mRNA was reverse transcribed to cDNA using a First Strand Kit (Invitrogen, Carlsbad, USA). The qRT-PCR was carried out with a QuantiTect SYBR Green RT-PCR kit (Qiagen, Hilden, Germany). Two microliters of total cDNA from each sample were amplified in a $25-\mu \mathrm{L}$ reaction mixture. The cycling conditions were as follows: denaturation at $65^{\circ} \mathrm{C}$ for $5 \mathrm{~min}$, snap cooling at $4^{\circ} \mathrm{C}$ for $1 \mathrm{~min}, 42^{\circ} \mathrm{C}$ for $50 \mathrm{~min}$, and finally at $72^{\circ} \mathrm{C}$ for $15 \mathrm{~min}$. The expression of the target gene was normalized to that of the glyceraldehyde-3-phosphate dehydrogenase (GAPDH). The relative gene expression was calculated using the $2^{\Delta \mathrm{CT}}$ formula.
Rat-specific primers were used for Tnmd, $S c x$ and GAPDH as follows: 5'-CCATGCTGGATGAGAGAGGTTAC-3' (forward) and 5'CACAGACCCTGCGGCAGTA-3' (reverse) for Tnmd; 5'-AACACGGCCTTCACTGCGCTG-3' (forward) and 5'-CAGTAGCACGTTGCCCAGGTG-3' (reverse) for Scx; 5'TGACTCTACCCACGGCAAGTTCAA-3' (forward) and 5'-ACGACATACTCAGCACCAGCATCA-3' (reverse) for GAPDH.

\section{Statistical analysis}

The data is presented as mean \pm standard deviation (SD). One-way analysis of variance (ANOVA) with the StudentNewman-Keuls test was used for multiple comparisons. Statistical analyses were carried out with SPSS v. 11.0 statistical package (IBM Corp., Armonk. USA). All p-values $<0.05$ were accepted as statistically significant.

\section{Results}

\section{Proliferative capacity of tendon stem cells}

The proliferation of TSCs was determined by PDT. The young TSCs grew faster than the aged TSCs when cultured on a plastic surface. Decellularized extracellular matrix from young and aged donors enhanced the proliferation of aged TSCs. It was observed that the PDT of TSCs was significantly lower in the aged TSC + young DECM and aged TSC + aged DECM groups compared to the aged TSC group (Fig. $1 ; \mathrm{p}<0.05$ ). These findings indicated that aging can decrease the proliferation of TSCs and that this feature could be altered by exposure to DECM. Unexpectedly, we found that there was no significant difference in the PDT of aged TSCs after culturing on young DECM and aged DECM.

\section{Senescence-associated $\beta$-galactosidase activity assay}

There was significantly higher $\beta$-galactosidase activity in aged TSCs compared to young TSCs when cultured on plastic (Fig. 2; p < 0.05), indicating that the $\beta$-galactosidase activity increased with age. Aged TSCs cultured on young DECM exhibited significantly lower levels of $\beta$-galactosidase activity when compared to those cultured on aged DECM or on plastic (Fig. 2; $\mathrm{p}<0.05)$.

\section{Stem cell marker expression}

Immunofluorescence staining for Oct-4 and SSEA-1 showed that the fluorescent density of stem cell markers was more extensive in young TSCs (Fig. 3,4). There were significant differences in the percentage of positive cells among the 2 groups (Fig. 5; p < 0.05). These findings 


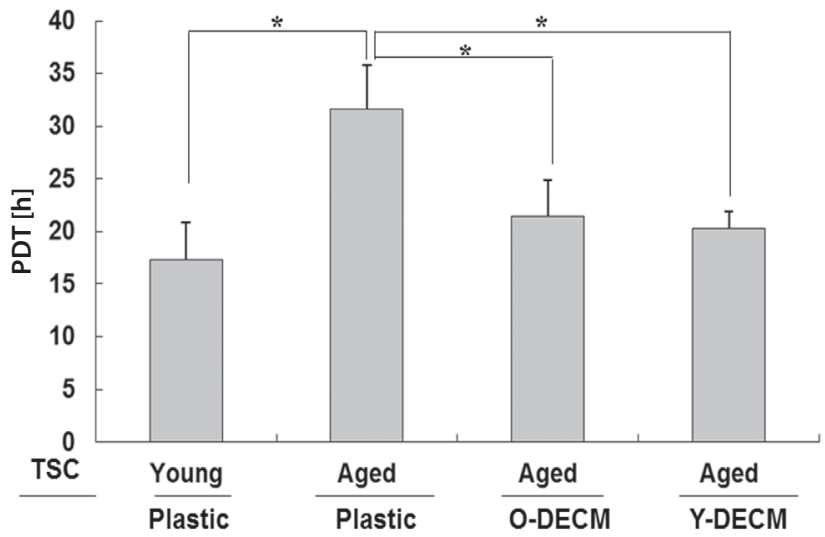

Fig. 1. The population doubling time (PDT) of TSCs on plastic, Y-DECM and O-DECM. The PDT of aged TSCs on plastic was significantly higher when compared to other groups

* $\mathrm{p}<0.05 ; \mathrm{Y}$-DECM - young decellularized extracellular matrix;

O-DECM - old decellularized extracellular matrix; TSCs - tendon stem cells.

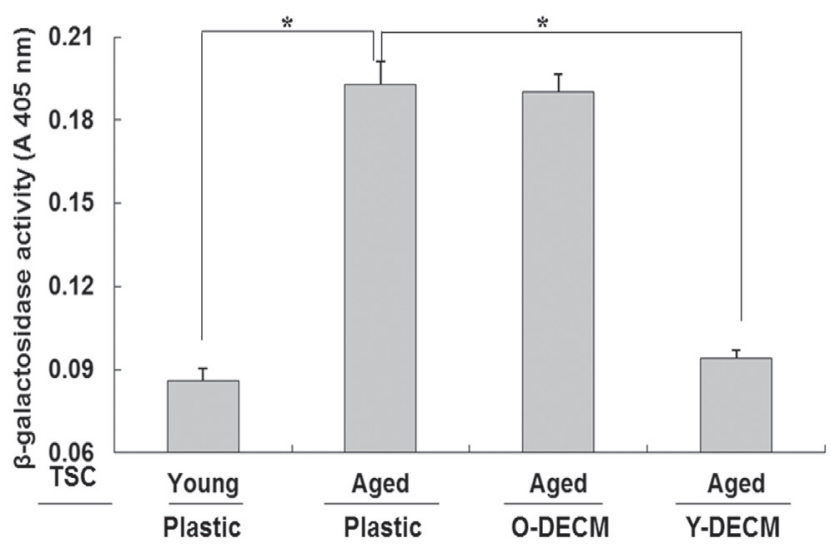

Fig. 2. The senescence-associated $\beta$-galactosidase activities of TSCS in different groups. The Y-DECM significantly decreased the $\beta$-galactosidase activity of aged TSCs

* $\mathrm{p}<0.05$; Y-DECM - young decellularized extracellular matrix;

O-DECM - old decellularized extracellular matrix; TSCs - tendon stem cells.

suggest that the decreased stem cell marker expression in aged TSCs may contribute to reduced stemness with age. After being cultured on young DECM, the expressions of Oct- 4 and SSEA- 1 by aged TSCs were more extensive than those expressed by aged TSCs cultured on aged DECM or on plastic.

\section{Tendon-related marker expression}

The results indicated that the expression of tendon lineage-specific genes, Tnmd and $S c x$, were lower in aged TSCs than in young cells (Fig. 6; $\mathrm{p}<0.05$ ). It was also found that expressions of Tnmd and Scx were significantly enhanced in the aged TSC + young DECM group when compared to that of the aged TSCs cultured on aged DECM or on plastic (Fig. 6; $\mathrm{p}<0.05$ ).
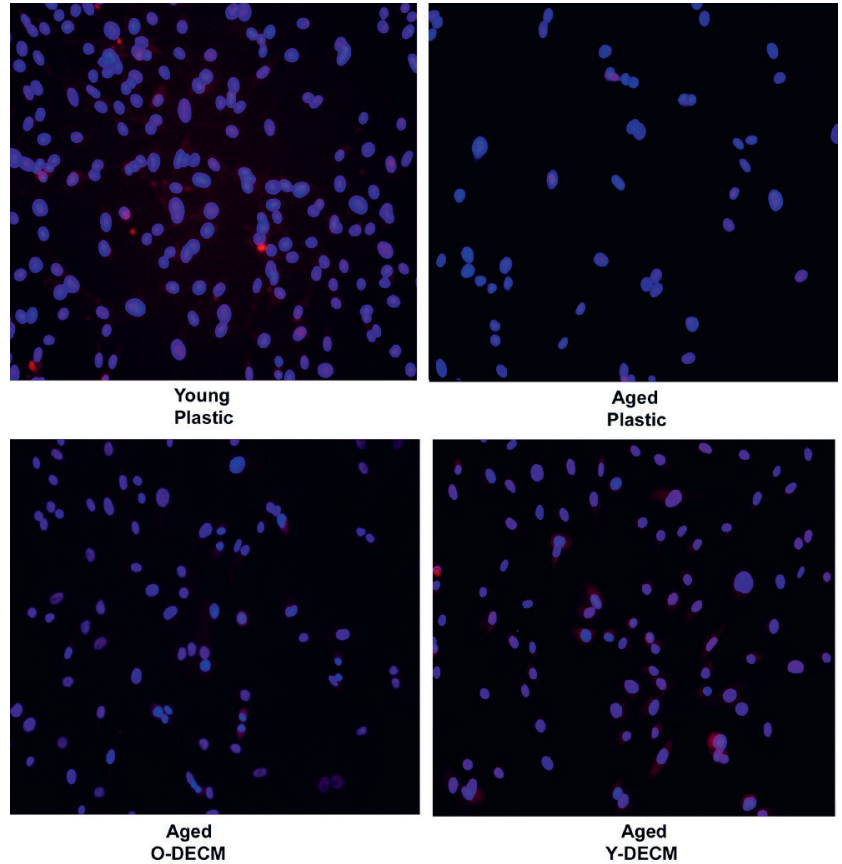

Fig. 3. The expression of octamer-binding transcription factor 4 (Oct-4) was evaluated by immunofluorescence assay. The density of Oct-4 in young TSC $s$ was higher than in aged TSCs. After being cultured on the Y-DECM, the expression of Oct- 4 by aged TSCs was upregulated. Original magnification is $\times 200$

Y-DECM - young decellularized extracellular matrix; O-DECM - old decellularized extracellular matrix; TSCs - tendon stem cells.
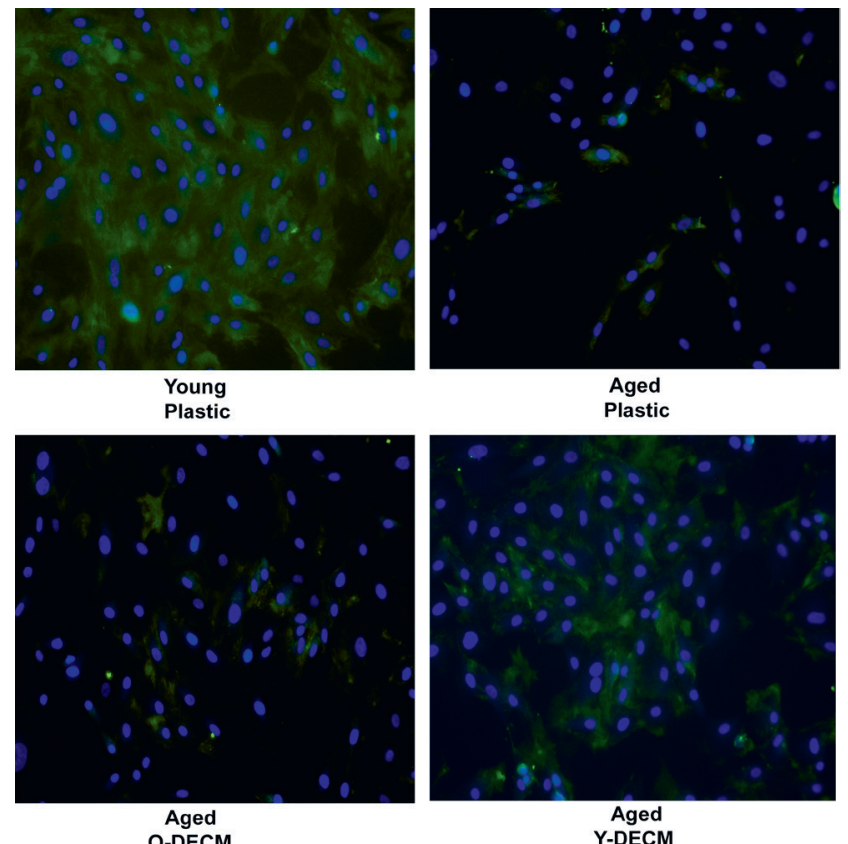

Fig. 4. The expression of stage-specific embryonic antigen-4 (SSEA-4) was evaluated by immunofluorescence assay. The density of SSEA-1 in young TSCs was higher than in aged TSCs. After being cultured on the Y-DECM, the expression of SSEA-1 by aged TSC 5 was upregulated. Original magnification is $\times 200$

Y-DECM - young decellularized extracellular matrix; O-DECM - old decellularized extracellular matrix; TSCs - tendon stem cells. 
A

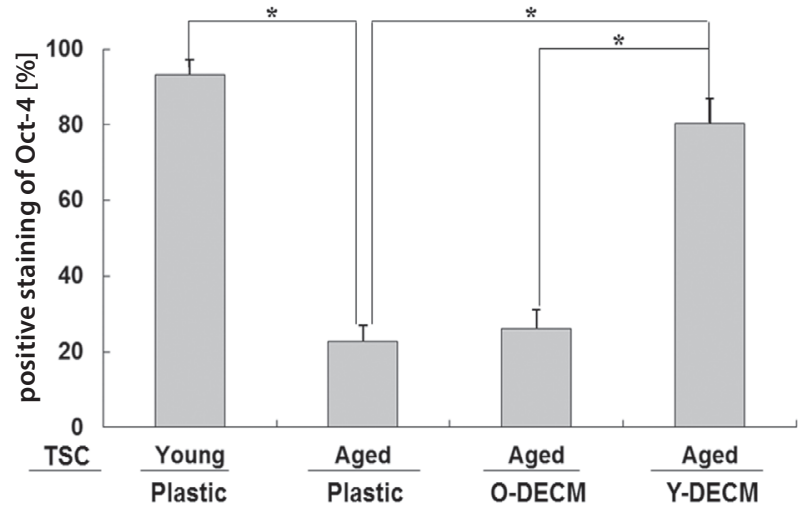

B

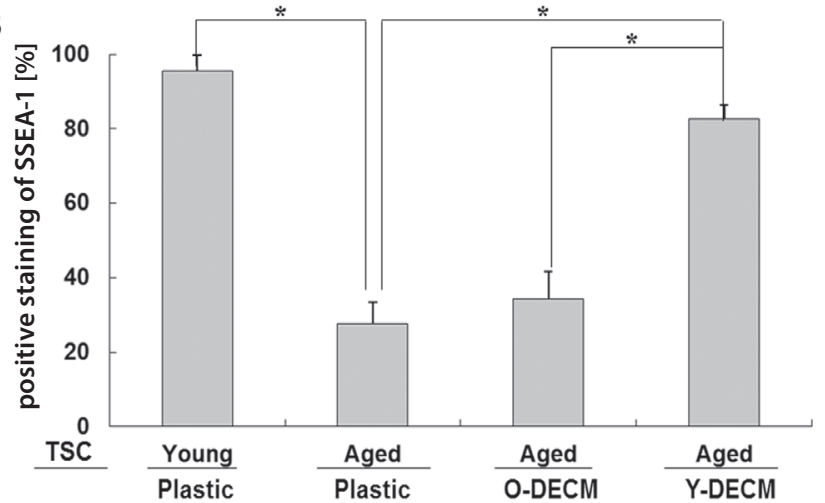

Fig. 5. The percentage of stem cell marker-positive cells. There were significant differences in the percentage of stem cell marker-positive cells among young and aged TSCs. After being cultured on the Y-DECM, the percentages of octamer-binding transcription factor 4 (Oct-4) (A) and stage-specific embryonic antigen-4 (SSEA-4) (B) in aged TSC s were increased

* $\mathrm{p}<0.05$; Y-DECM - young decellularized extracellular matrix; O-DECM - old decellularized extracellular matrix; TSCs - tendon stem cells.

A

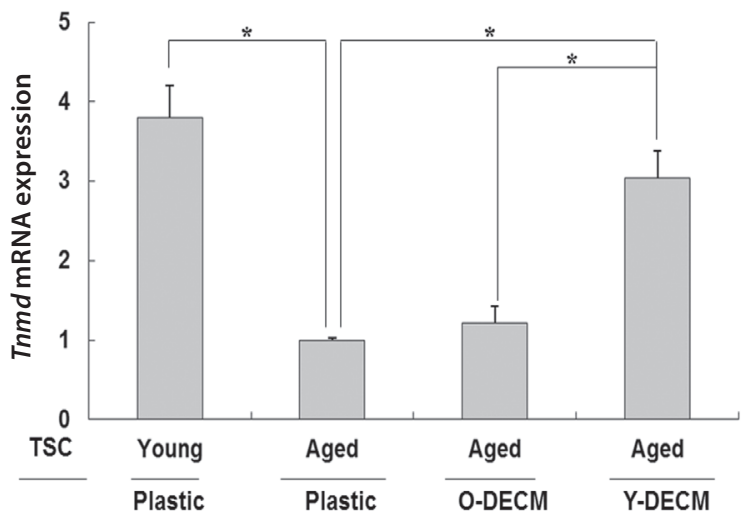

B

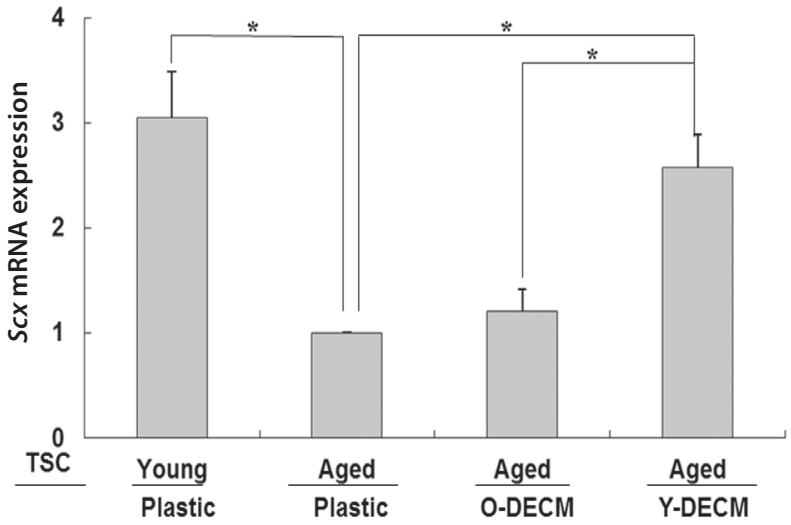

Fig. 6. The expression of tenocyte-related genes was evaluated by quantitative reverse transcription polymerase chain reaction (qRT-PCR). The mRNA of Tnmd (A) and Scx (B) were significantly upregulated in TSCs after treatment with Y-DECM. The expression level was normalized to that of glyceraldehyde-3-phosphate dehydrogenase (GAPDH).

${ }^{*} \mathrm{p}<0.05$; Y-DECM - young decellularized extracellular matrix; O-DECM - old decellularized extracellular matrix; TSCs - tendon stem cells.

\section{Discussion}

Tendon stem cells can form a colony, self-renew and differentiate into multiple cell lineages. ${ }^{7,8}$ Moreover, as tendonspecific stem cells, they can differentiate into tenocytes by default. ${ }^{16}$ As a result of these capabilities, TSCs play an important role in the continuous maintenance and regeneration of injured tendons. ${ }^{9,10}$ Recently, it was reported that the number and function of TSCs decrease with advancing age, which may affect the structure and function of tendon tissue. ${ }^{5}$ It remains to be determined whether these agerelated changes can be reversed. Thus, we examined whether properties of aged TSCs would be altered by exposure to ECM produced by young TSCs.

In this study, we showed that the deleterious effect of aging on the TSCs was remarkable when the cells were cultured on plastic. As previously reported, a higher proliferation potential of TSCs from young donors was observed when compared to that of aged donors. ${ }^{5}$ It also corroborated the results obtained from periodontal ligament stem cells and tenocytes. ${ }^{6,17}$ These results suggest a significant impact of aging on TSCs, ligament stem cells and tenocytes in terms of proliferative activity and differentiation potential. In addition to the effect of donor age on the proliferation of TSCs, we investigated its effects on the senescence-associated $\beta$-galactosidase activity. As a marker of aging, increased $\beta$-galactosidase activity was observed in aged TSCs. However, the surface expressions of Oct-4 and SSEA-4 in aged TSCs were downregulated in our study. These may reduce the pool of TSCs for the regeneration of aged tendons after injury. It was also observed that tenogenic activity was reduced in aged donors. This result was supported by the tendon lineagespecific gene expression, which revealed age-related declines in the expression of Tnmd and Scx. The loss of tenogenic differentiation potential with aging was also reported in a previous study. ${ }^{5}$ This implies that there are fewer stem cells with tenogenic potential in aged donors than in younger donors.

Tendon stem cells are surrounded by ECM in tendon tissue, which form a microenvironment or niche. Recent studies have demonstrated the importance of extrinsic ECM 
on the rejuvenation of stem cells. ${ }^{13,18}$ For example, aged mesenchymal stem cells can continue to function without any decline in function when cultured on a young ECM. ${ }^{18}$ These findings made us question whether aged TSCs can be influenced by the ECM obtained from young donors. In this study, we successfully developed DECM scaffold using TSCs in vitro. It was found that DECM prepared from young and aged TSCs significantly promoted the proliferation of aged TSCs. It indicated that DECM of TSCs might provide a proper microenvironment that can mimic the niche that TSCs reside in. This feature of ECM obtained from TSCs is similar to a recent finding that ECM deposited by human bone marrow stromal cells (BMSCs) facilitates the proliferation of BMSCs. ${ }^{19}$

Our study also provided evidence that young DECM preserved the stem cell properties of aged TSCs. We measured several stem cell markers such as Oct-4 and SSEA-4; Oct-4 is essential for maintaining undifferentiated pluripotent stem cells, while SSEA-4, originally identified as an early embryonic glycolipid antigen, is a molecule characteristic of undifferentiated pluripotent human stem cells. ${ }^{20,21}$ Our data revealed that the proportion of those positive cells was significantly higher in aged TSCs when treated with young DECM. Moreover, the senescence-associated $\beta$-galactosidase activity of aged TSCs was decreased by young DECM. It was suggested that high quality of stem cells from aged TSCs was enriched by exposure to a young DECM.

In parallel with determining the effects of young DECM on cell proliferation and stemness, we also examined the effect of young DECM on the tenogenic differentiation potential of aged TSCs. Young DECM had significantly increased the mRNA expression of tenogenic markers, such as Tnmd and $S c x$. Our data provided evidence that young DECM enhanced the tenogenic differentiation potential of aged TSCs. Together, this data demonstrates that young DECM is capable of rejuvenating the altered biological activities of aged TSCs.

It was reported that compositions of ECM deposited by young and old cells are different. ${ }^{18}$ Another study also suggested that tissue-specific stem cells could retain their intrinsic capacity even when old, but the aged niche in which stem cells reside inhibit the signal pathway activation of these cells. ${ }^{22}$ Thus, aged TSCs, as a surrounding environment, may be the major determinant causing aging of cells. ${ }^{18}$ The composition of DECM from young or aged TSCs should be different. However, we did not compare the composition of these 2 ECMs in this study. Moreover, a signal mechanism might contribute to the rejuvenation of aged TSCs by young DECM. Further studies are required to investigate these problems.

\section{Conclusions}

We have shown that the aging process induces senescence and a decline in the proliferation, stemness and tenogenic differentiation of TSCs. Moreover, the impaired capacity of aged TSCs can be improved by exposure to young DECM, thereby improving the effectiveness of aged TSCs. The positive results of our study suggest that the use of young DECM could be promising in the prevention and treatment of age-dependent tendon disorders.

\section{References}

1. Couppé $C$, Hansen $P$, Kongsgaard M, et al. Mechanical properties and collagen cross-linking of the patellar tendon in old and young men. J Appl Physiol (1985). 2009;107(3):880-886.

2. Kostrominova TY, Brooks SV. Age-related changes in structure and extracellular matrix protein expression levels in rat tendons. Age (Dordr). 2013;35(6):2203-2214.

3. Klatte-Schulz F, Pauly S, Scheibel M, et al. Influence of age on the cell biological characteristics and the stimulation potential of male human tenocyte-like cells. Eur Cell Mater. 2012;24:74-89.

4. Dudhia J, Scott CM, Draper ER, Heinegård D, Pitsillides AA, Smith RK. Aging enhances a mechanically-induced reduction in tendon strength by an active process involving matrix metalloproteinase activity. Aging Cell. 2007;6(4):547-556.

5. Zhou Z, Akinbiyi T, Xu L, et al. Tendon-derived stem/progenitor cell aging: Defective self-renewal and altered fate. Aging Cell. 2010;9(5):911-915.

6. Tsai WC, Chang HN, Yu TY, et al. Decreased senescence-inhibited gene and up-regulation of p27. J Orthop Res. 2011;29(10):1598-1603.

7. Tan Q, Lui PP, Rui YF. Effect of in vitro passaging on the stem cellrelated properties of tendon-derived stem cells-implications in tissue engineering. Stem Cells Dev. 2012;21(5):790-800.

8. Bi Y, Ehirchiou D, Kilts TM, et al. Identification of tendon stem/progenitor cells and the role of the extracellular matrix in their niche. Nat Med. 2007;13(10):1219-1227.

9. Chen L, Dong SW, Liu JP, Tao X, Tang KL, Xu JZ. Synergy of tendon stem cells and platelet-rich plasma in tendon healing. J Orthop Res. 2012;30(6):991-997.

10. Ni M, Rui YF, Tan Q, et al. Engineered scaffold-free tendon tissue produced by tendon-derived stem cells. Biomaterials. 2013;34(8):2024-2037.

11. Zhang J, Li B, Wang JH. The role of engineered tendon matrix in the stemness of tendon stem cells in vitro and the promotion of tendon-like tissue formation in vivo. Biomaterials. 2011;32(29):6972-6981.

12. Pei M, He F. Extracellular matrix deposited by synovium-derived stem cells delays replicative senescent chondrocyte dedifferentiation and enhances redifferentiation. J Cell Physiol. 2012;227(5):2163-2174.

13. He F, Pei M. Rejuvenation of nucleus pulposus cells using extracellular matrix deposited by synovium-derived stem cells. Spine. 2012;15(37):459-469.

14. Li J, Pei M. Optimization of an in vitro three-dimensional microenvironment to reprogram synovium-derived stem cells for cartilage tissue engineering. Tissue Eng Part A. 2011;17(5-6):703-712.

15. Rui YF, Lui PP, Wong YM, Tan Q, Chan KM. Altered fate of tendonderived stem cells isolated from a failed tendon-healing animal model of tendinopathy. Stem Cells Dev. 2013;22(7):1076-1085.

16. Zhang J, Wang JH. Characterization of differential properties of rabbit tendon stem cells and tenocytes. BMC Musculoskelet Disord. 2010;11:10. doi: 10.1186/1471-2474-11-10

17. Zheng W, Wang S, Ma D, Tang L, Duan Y, Jin Y. Loss of proliferation and differentiation capacity of aged human periodontal ligament stem cells and rejuvenation by exposure to the young extrinsic environment. Tissue Eng Part A. 2009;15(9):2363-2371.

18. Sun Y, Li W, Lu Z, et al. Rescuing replication and osteogenesis of aged mesenchymal stem cells by exposure to a young extracellular matrix. FASEB J. 2011;25(5):1474-1485.

19. Pei $M, H e F, K i s h ~ V L$. Expansion on extracellular matrix deposited by human bone marrow stromal cells facilitates stem cell proliferation and tissuespecific lineage potential. Tissue Eng Part A. 2011;17(23-24):3067-3076.

20. Pesce M, Scholer HR. Oct-4: Gatekeeper in the beginnings of mammalian development. Stem Cells. 2001;19(4):271-278.

21. Gang EJ, Bosnakovski D, Figueiredo CA, Visser JW, Perlingeiro RC. SSEA-4 identifies mesenchymal stem cells from bone marrow. Blood. 2007;109(4):1743-1751.

22. Conboy IM, Conboy MJ, Wagers AJ, Girma ER, Weissman IL, Rando TA. Rejuvenation of aged progenitor cells by exposure to a young systemic environment. Nature. 2005;433(7027):760-764. 